Historic, Archive Document

Do not assume content reflects current scientific knowledge, policies, or practices. 



\title{
Fall 1898
}

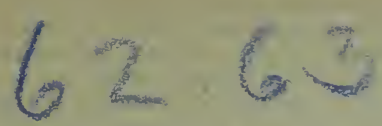

\section{PINEHURST NURSERIES,} 111

\author{
PINEHURST, N. C., U. S. A.
}

Broad-Ireaved Everorreens, OrnamentaI Trees, Shrubs and Vines,

\section{NATIVE WOODY and HERBACEOUS PLANTS AND SEEDS.}

D.

PLf ur stock has been regularly inspected by the North Carolina State Entomologist, who has certified SEE that it gave no indication of any species of insects designated as dangerous pests by the North Carolina Commission for Controlling Crop Pests, and that it was healthy in every respect. 
Address all letters, telegrams and telephones to PINEHURST NURSERIES, PINEHURST, N. C. Our freight office is Pinehurst. Express will be forwarded from Southern Pines, N. C., from where an electric railroad runs to Pinehurst.

TERMS: Cash or satisfactory reference. Remit by express or postoffice money order, registered letter, bank-draft, or check.

C. O. D. SHIIFMENTS by express made only upon prepayment of one-half the amount of the order. Charges for collection will be added to bill.

REDUC'TIONS: 5 plants of one kind are sold at 10-rates; 50 at 100-rates; 500 at 1000-rates. Special prices for larger quantities upon application. Packing charged at cost.

For extra strong especially selected specimens higher prices in proportion will be charged.

SHIPPING DIRECTIONS: Precise directions as to kind of conveyance (mail, express, freight) and route are desired, or we shall send at our own discretion.

COLLECTED SHRUBBERY: We forward strong, well rooted specimens only, the tops of which we generally cut back to save freight, if we are not especially instructed to the contrary. Should collected plants arrive in somewhat dried up condition as a result of long distance shipments or delay in transportation, cover the roots with damp moss and water them repeatedly before planting.

SHIPIIENTS OF SHRUBBERY are made from October 1st to about April 15th without hardly any interruption. Since spring begins here in some years unusually early and prevents any further digging, we advise to send orders for woody plants as much as possible in fall.

HERBACEOUS PLANTS: Quite a number of the perennials offered we have now in nursery rows, while others we shall collect after receipt of orders. Some kinds can be found only when in bloom. Orders on such will be filled in due season. Purchaser will in every case be notified immediately after receipt of order of time of shipment.

SEEDS: We warrant all our seeds fresh of last crop and take every precaution to have them perfectly true to name, but we give no warranty, express or implied, as to name, description, or any other matter, of any seeds we send out. If the purchaser does not accept the seeds on these terms he must. return them at once and the money which has been paid us for them will be refunded.

C. D. BENBOWV, OTTO KATZENSTEIN, Resident Manager for James W. Tufts, of Boßton, Mass. SUPERINTENDENT OF NURSERIES. Pinehurst, N. C., September 1st, 1898. 


\section{JUST A FEW WORDS.}

We desire to give expression to our sincerest thanks for the ready response which the offer of our plants and seeds has had during the past season. It has encouraged us to increase our capacities for prompt handling of orders and to enlarge our stock considerably by adding many interesting new species to our collections. We will just mention here that for instance the Fothergilla alnifolia, which had disappeared so long from American and most foreign nursery lists, has been found again by us in sufficient quantities to enable us to offer plants and seed of it at very moderate rates. A perusal of the following pages will show a number of equally valuable additions not only of woody but also herbaceous plants. Quite a number of the latter are gems of the highest merit in every way, but have hardly ever before found their way into nursery lists. Our Pinchurst Violet has even surpassed our fondest anticipations by the way it has been taken up. We cannot indeed say too much in praise of this splendid all-around valuable plant, very large quantities of which have been sent out. Our large stock of this violet looks as healthy and thrifty as possible.

In response to many inquiries we wish to emphasize here also that the majority of the plants offered by us will prove hardy and well adapted to Northern and European gardens. We shall, however, always be glad to assist in the selection of suitable collections for any given location.

We wish to thank again our patrons for past farors and solicit trial orders from all others which will ał ways have our promptest and most careful attention. 


\section{WOODY PLANTS.}

E, Evergreen. D, Deciduous. 1/2E, Nearly Evergreen. t, tree. s, shrub. v, trailer or vine.

Col., collected plants. S, Seedling plants. C, In Cutting bed. All others are heavy nursery grown. Collected plants can be furnished in larger sizes than quoted at proportionate prices.

NAIIE. Size.

E s Abelia floribunda. $-2-3 \mathrm{ft}$.

D t Acer rubrum-Red Maple 3-4 ft. 2-3 col.

D t Acer saccharinum-Sugar Maple 1-3 ft. col. $\frac{1}{2} \mathrm{E}$ r Akebia quinata-Five-fingered Akebia $-1 \frac{1}{2}-2 \mathrm{ft}$.

$3-4 \mathrm{ft}$.

D t Aíbizzia julíbrissin-Mimosa $3-6$ it.

D v Ampelopsís quinquefolia-Tirginia Creeper 9-12 in.

D s Andromeda mariana-Staggerbush___________-2-3 it. col.

E s ANDROIVEDA NITIDA-Fetterbush $1-2 \mathrm{ft}$.

$2-3 \mathrm{ft} . \mathrm{col}$.

D s Baccharis halimifolia-Groundsel 3 it.

Es Berberis ilicifolia-Holly-leaved Barberry $1-1 \frac{1}{2} \mathrm{ft}$.

D s " thunbergii-Thunberg's Barberry $6-9$ in.

D s " vulgaris - Common Barberry _._._._. 11 11 $2 \mathrm{ft}$.

E v Bignonía capreolata-Crossvine

D v " radicans-Trumpet-rine $2-3 \mathrm{ft} . \mathrm{col}$. 3- $4 \mathrm{ft}$. $2-3 \mathrm{ft}$. col.

D e Broussonetia papyrifera-Paper Mulberry $4-6 \mathrm{ft}$.

Es Buxus sempervirens-Evergreen Box 6-9 in.

D s Calycanthus floridus-Spice Shrub 2-3 ft. col.

Price of Plants. Each $\quad 10 \quad 100 \quad 1000$ $\$ 030 \$ 250$

$25 \quad 100 \$ 400 \$ 2500$ $\begin{array}{lll}50 & 200 \quad 1200\end{array}$ $150 \quad 500$

$\begin{array}{llll}15 & 75 & 500 & 2000\end{array}$

$25 \quad 100 \quad 700 \quad 5000$

$20 \quad 75 \quad 500$

$15 \quad 75 \quad 300$

$15 \quad 75 \quad 400 \quad 1200$

$25 \quad 200 \quad 600 \quad 2500$ $100 \quad 300 \quad 1000$

$25 \quad 150$

$35 \quad 250$

$10 \quad 60 \quad 300$

15 200

75

$$
3
$$

3

$75 \quad 300 \quad 2000$

$25 \quad 125 \quad 600$

$15 \quad 100 \quad 350 \quad 2000$

$25 \quad 100 \quad 500$

$70 \quad 300$
Price of Seeds. Oz. Lb.

$\$ 015 \$ 080$

$15 \quad 75$

$15 \quad 75$

$30 \quad 225$

$15 \quad 100$

$15 \quad 120$

$10 \quad 50$ 
TAME:

Ds Castanea pumila-Chinguapin Chestnut $2-3 \mathrm{ft}$.

$2-3 \mathrm{ft}$. col.

D s Ceanothus americanus-Jersey Tea 2 it col.

E t Cedrus atlantica-Mit. Atlas Cedar $2 \mathrm{ft}$.

E $t$ " deodara-Deodar Cedar $21,-3 \mathrm{ft}$.

E t Cephalotaxus fortunei-Fortune's Yew $1-1 \frac{1}{2} \mathrm{ft}$.

D t Cercis canadensis-Red Bud $1-3 \mathrm{ft}$.

$2-3 \mathrm{ft} . \mathrm{col}$.

E t Chamaecyparis thuyoides-White Cedar $3 \mathrm{ft}$.

$2-3 \mathrm{ft}$. col.

Ds Chionanthus virginica-White Fringe $1-2 \mathrm{ft}$. 1-2 ft. col.

D s Cladrastis tinctoria-Yellow Wood 1-3 ft. col.

D v Clematis crispa-Blue Jessamine strong col.

D s Clethra alnifolia_Sweet Pepper Bush $-2-3 \mathrm{ft}$.

2-3 ft. col.

${ }_{2}^{1} \mathrm{E}$ s Cliftonia ligustrina-Cliftonia $2-3 \mathrm{ft}$. col.

D s Cornus florida-White flowering Dogwood $1-2 \mathrm{ft}$.

$2-3 \mathrm{ft}$.

$3-5 \mathrm{ft}$.

$2-3$ it. col.

3-5 ft. col.

Es Cotoneaster microphylla_Evergreen Cotoneaster _-_._1-2 ft.

$\mathrm{E} s$ Crataegus dentata-Tooth-leaved Thorn -_._-_-_-_2-3 ft.

$\mathrm{E} s \quad$ " glabra _-_

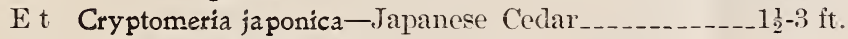

E t Cupressus fastigiata-Italian Cypress _._._.........-2-3 ft.
Price of I'lants.

Price of Seerls.

\begin{tabular}{|c|c|c|c|c|c|}
\hline \multirow{2}{*}{\multicolumn{6}{|c|}{ 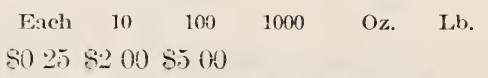 }} \\
\hline & & & & & \\
\hline & 100 & & $\$ 1200$ & $\$ 010$ & 8060 \\
\hline 20 & 75 & +00 & 1500 & 10 & 7 \\
\hline 35 & 200 & & & & \\
\hline 35 & 200 & 1200 & & & \\
\hline 25 & 200 & & & & \\
\hline \multirow[t]{2}{*}{25} & 120 & 500 & 2500 & 25 & 100 \\
\hline & 75 & 300 & 1500 & & \\
\hline \multirow[t]{2}{*}{25} & 150 & $6(10)$ & & 15 & 100 \\
\hline & 75 & 300 & 2000 & & \\
\hline \multirow[t]{2}{*}{25} & 125 & 900 & & 15 & 100 \\
\hline & 75 & 600 & 2500 & & \\
\hline 15 & 75 & 400 & & & \\
\hline 20 & 100 & 500 & 4500 & & \\
\hline \multirow[t]{2}{*}{25} & 100 & 300 & & & \\
\hline & 75 & 250 & 1200 & 15 & 100 \\
\hline 20 & 100 & 400 & 1200 & 20 & 100 \\
\hline 10 & 75 & +00 & 1800 & 10 & 40 \\
\hline 15 & 100 & 500 & 2000 & & \\
\hline \multirow[t]{3}{*}{25} & 150 & 900 & 4000 & & \\
\hline & 50 & 300 & 1200 & & \\
\hline & 75 & 500 & 2000 & & \\
\hline 25 & 200 & 1000 & & & \\
\hline 50 & 300 & & & & \\
\hline 50 & 300 & & & & \\
\hline 25 & 200 & & & & \\
\hline 25 & 200 & & & & \\
\hline
\end{tabular}




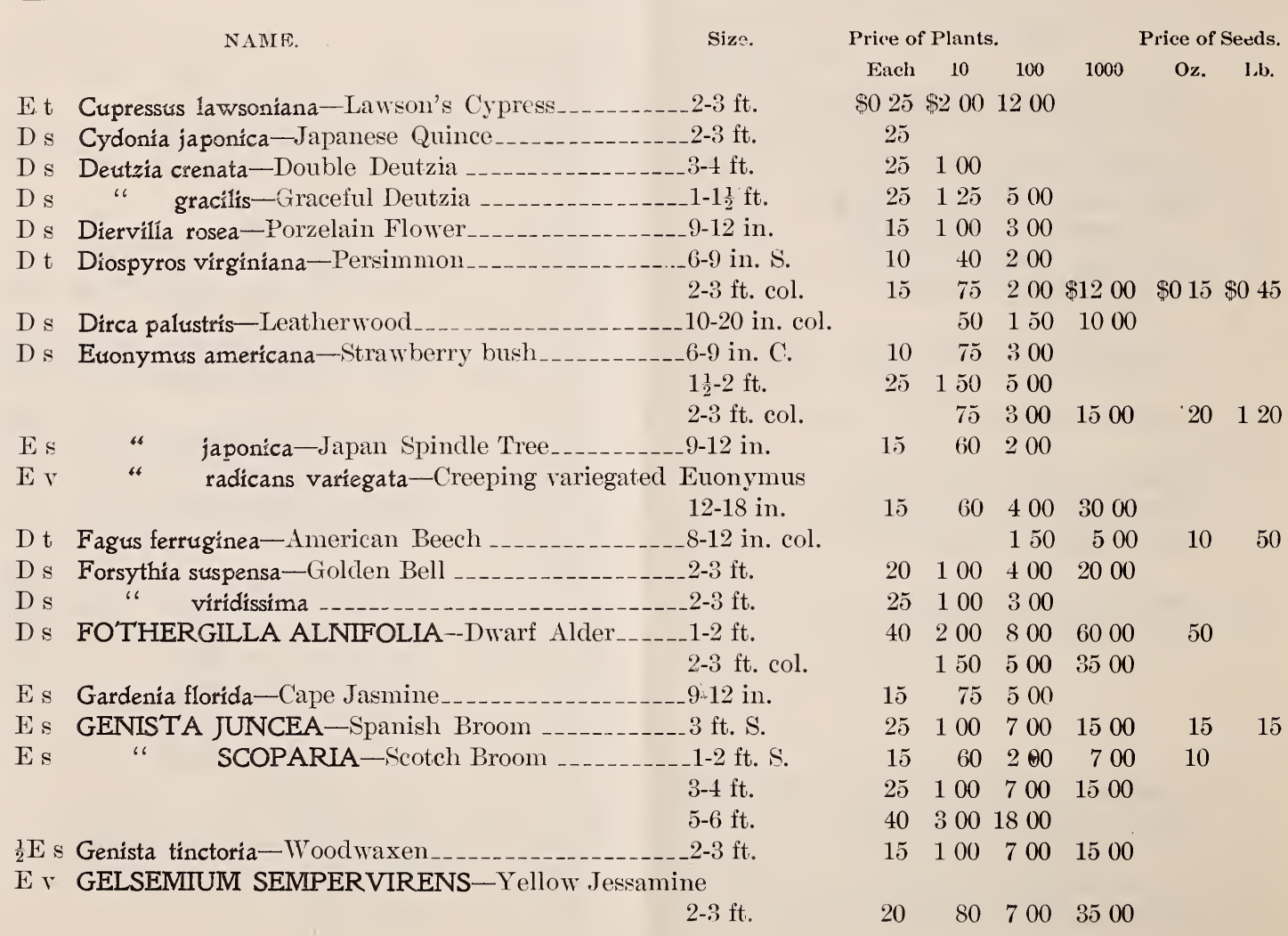


NAME.

Size.

E $\mathrm{v}$ GELSEMIUIM SEMIPERVIRENS-Yellow Jessamine

$$
3-4 \mathrm{ft} \text {. }
$$

strong col.

light col.

E t Gordonia lasianthers-Loblolly Bay $4 \mathrm{ft}$.

3-5 ft. col.

D s Hamamelis virginica-Witch Hazel $1 \frac{1}{2}-2 \frac{1}{2} \mathrm{ft}$.

E r Hedera helix-English Iry $1 \frac{1}{2}-3 \mathrm{ft}$. strong

D s Hibiscus syriacus-Althaea $2-3 \mathrm{ft}$.

Es Hypericum calycinum-St. John's Wort 1 yr.

E s HYPERICUM MOSERIANUM

E s Ilex dahoon-Dahoon Holly $1 \mathrm{yr}$. $-1 \frac{1}{2}-3 \mathrm{ft}$. 2-3 ft. col.

E s " glabra-Inkberry $1-2 \mathrm{ft}$. $1 \frac{1}{2}-2 \frac{1}{2} \mathrm{ft}$. col.

E t ILEX OPACA-American Holly (Prices for fruited specimens upon application.) $1-2 \mathrm{ft}$. $2-3 \mathrm{ft}$. 1-3 ft. col.

D s Ilex verticillata-Deciduous Holly -2-3 it. col. $\frac{1}{2} \mathrm{E} s$ JASMINUM NUDIFLORUMI-Yellow Jasmine 9-12 in. $1 \frac{1}{2}-2 \mathrm{ft}$. $2-5 \mathrm{ft}$.

D t Juglans nigra-Black Walnut -1-2 ft.

$\mathrm{E} t$ Juniperus virginiana-Red Cedar $2-3 \mathrm{ft}$. 1-2 ft. col.

D s Lagerstroemia indica-Crape Myrtle $1-2 \mathrm{ft}$.
Price of Plants.

Price of Seeds.

$\begin{array}{llllll}\text { Each } & 10 & 100 & 1000 & \text { Oz. } \quad \text { Lb. }\end{array}$

$\$ 025 \$ 100 \$ 800 \$ 4000$

$75 \quad 500 \quad 2000$

$40 \quad 200 \quad 800$

100

$40 \quad 3 \quad 00 \quad 1800 \quad 12000 \quad \$ 050 \$ 500$

$25 \quad 125 \quad 700$

$20 \quad 100 \quad 400 \quad 2000$

$\begin{array}{llll}15 & 100 & 400\end{array}$

$25 \quad 125 \quad 700$

$25 \quad 125 \quad 700$

$25 \quad 1501000$

$\begin{array}{lllll}75 & 500 & 2000 & 40 & 400\end{array}$

$25 \quad 150 \quad 1000 \quad 4000$

$75 \quad 500 \quad 2000$

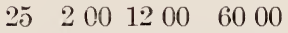

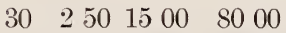

$\begin{array}{llll}150 & 800 & 4000\end{array}$

$15 \quad 120$

$15 \quad 75 \quad 500 \quad 2000$

$10 \quad 60 \quad 400 \quad 3000$

$15 \quad 80 \quad 500 \quad 3500$

$25 \quad 125 \quad 700 \quad 5000$

$\begin{array}{llllll}25 & 1 & 50 & 10 & 00\end{array}$

$25 \quad 1251000$

$\begin{array}{llllll}10 & 75 & 600 & 3500 & 15 & 75\end{array}$

$25 \quad 200 \quad 30$ 
NAME.

Size.

$\begin{array}{ll}\text { E s LAURUS LA TIFOLIA-Broad-leaved Lamel } & \text { L } \\ \text { E s Kalmia latifolia_-Mountain Laurel } & 1 \frac{1}{2}-2 \mathrm{ft} .\end{array}$

E s Leucothoe catesbaei-Dog Jaurel _._._._._._._._.

$1 \frac{1}{2}-2$ ft. col.

$\mathrm{Es}$

E s IIGUSTRUMM CHIN

"racemosa

$1 \mathrm{ft}$.

9-12 in. C.

Dwarf Chinese Privet _._.12-18 in.

$18-2+$ in.

$2-3 \mathrm{ft}$.

$3-5 \mathrm{ft}$

E s Ligustrum lucidum

3-6 in. S.

$\frac{1}{2} \mathrm{E}$ s LIGUSTRUM OVALIFOLIUM-California Privet_9-12 in. C.

$12-18$ in.

$18-24$ in.

$2-3 \mathrm{ft}$.

$3-5 \mathrm{ft}$.

$\frac{1}{2} \mathrm{E} s$ Limonia trifoliata-Hardy Lemon

9-12 in.

2-4 ft.

D t Liquidambar styraciflua-Sweet Gum

$2-4 \mathrm{ft}$. col.

D t Liriodendron tulipifera-Tulip Tree
2-4 ft. col.

Price of Plants.

Price of Seeds

$\begin{array}{llllll}\text { Each } & 10 & 100 & 1000 & \text { Oz. Lb. }\end{array}$

$\$ 035 \$ 250 \$ 1000$

$\begin{array}{lllllllll}15 & 100 & 500 & \$ 30 & 00 & \$ 0 & 25 & \$ 1 & 25\end{array}$

$25 \quad 120$

$125 \quad 500 \quad 2000$

$25 \quad 100 \quad 400$

$10 \quad 50 \quad 200 \quad 1000$

$\begin{array}{llll}10 & 60 & 300 & 1500\end{array}$

$15 \quad 75 \quad 400 \quad 2000$

$25 \quad 100 \quad 500 \quad 2500$

$40 \quad 300 \quad 1000$

$10 \quad 75 \quad 600$

$10 \quad 50 \quad 200 \quad 1000$

$\begin{array}{llll}10 & 60 & 300 & 1500\end{array}$

$15 \quad 75 \quad 400 \quad 2000$

$25 \quad 100 \quad 600 \quad 3000$

$\begin{array}{llll}30 & 150 & 800\end{array}$

$\begin{array}{lllll}20 & 100 & 600\end{array}$

$40 \quad 300$

$\begin{array}{lllllll}15 & 100 & 300 & 1500 & 35 & 200\end{array}$

$\begin{array}{lllllll}15 & 75 & 200 & 1000 & 15 & 60\end{array}$

E v LONICERA BRACHYPODA HALIEANA-Japanese Honeysuckle

\section{0,000 rates upon}

application.
$2-3 \mathrm{ft} . \mathrm{C}$.

$2-3 \mathrm{ft}$.

3-5 ft.

E v Lonicera brach. fol, aureo-reticulatis-Golden Honeysuckle

9-12 in.

$1 \frac{1}{2}-3 \mathrm{ft}$. $\begin{array}{llll}30 & 150 & 600\end{array}$

$15 \quad 50 \quad 300 \quad 1800$

$25 \quad 75 \quad 500 \quad 2500$
$15 \quad 75 \quad 400 \quad 2500$

$20 \quad 100 \quad 500 \quad 3000$ 
NAME.

Size.

E y Lonicera chinensis-Chinese Honeysuckle

2-3 ft. C.

$2-3 \mathrm{ft}$.

$3-4 \mathrm{ft}$.

D v " semperflorens-Woodbine 12 in.

$1-3 \mathrm{ft} . \mathrm{col}$.

Es Lycium europaeum

$3-4 \mathrm{ft}$.

D s Maclura aurantiaca-Osage Orange

$2-3 \mathrm{ft}$.

D t Magnolia acuminata-Cucumber Tree 1-2 ft. col.

$\frac{1}{2} \mathrm{E} \mathrm{t}$

glauca-Sweet Bay $1-2 \mathrm{ft}$. $2-3 \mathrm{ft}$. $3-4$ ft. col.

E t

4

grandíflora-Evergreen Magnolia 3-4 ft.

18-24 in.

D t " tripetala-Umbrella Tree 1-2 ft. col.

E s Mahonia aquifolium-Asheberry _................ $1 \frac{1}{2} \mathrm{ft}$.

$\mathrm{E} \mathrm{s}$ " japoníca _-_..._.

D t Melia azederach-Pride of China_________._._._. $3-4 \mathrm{ft}$. 4-6 ft.

D t “ umbraculifera-Texas Umbrella Tree_-2-3 ft.

E s Myrica cerifera-Bay Berry $2-3 \mathrm{ft}$. 2-3 ft. col.

Es " pumila-Dwarf Wax Myrtle 12-18 in. col.

D t Nyssa sylvatíca-Tupelo $2-3 \mathrm{ft}$. col. 3-5 ft. col.

E s Osmanthus ilicifolius $1-1 \frac{1}{2}$

D s Oxydendrum arboreum-Sourwood
$-2-3 \mathrm{ft}$.

$2-3 \mathrm{ft}$. col.
Price of Plants. Each

$$
\$ 040 \$ 200 \$ 1000
$$

$\$ 020 \quad 75 \quad 500 \quad 3000$

$25 \quad 100 \quad 600 \quad 4000$

$15 \quad 60 \quad 300$

$\begin{array}{lll}75 & 300 \quad 1200\end{array}$

$25 \quad 150$

$25 \quad 100 \quad 800$

$75 \quad 300 \quad 1000 \quad \$ 020 \$ 120$

$\begin{array}{lllllll}20 & 200 & 1000 & 7000 & 20 & 150\end{array}$

$\begin{array}{lllllll}25 & 2 & 25 & 12 & 00 & 90 & 00\end{array}$

$150 \quad 900 \quad 7000$

$\begin{array}{llll}50 & 475 & 4500\end{array}$

35300

$75 \quad 250$

$25 \quad 1501000$

25

25

3

$100 \quad 500 \quad 3000$

$15 \quad 75$

$\begin{array}{lllll}150 & 600 \quad 4000\end{array}$

$25 \quad 125 \quad 500$

$15 \quad 75$

$25 \quad 150 \quad 700 \quad 4000$

$100 \quad 500 \quad 2000$

$15 \quad 80$

$20 \quad 120$

$\begin{array}{lllll}20 & 100 & 500 & 3000\end{array}$

$\begin{array}{lllll}10 & 75 & 400 & 1500\end{array}$

$\begin{array}{lllll}15 & 100 & 600 & 1800\end{array}$

$15 \quad 50$

35

$25 \quad 200 \quad 800$

15

$100 \quad 400 \quad 2000$
$15 \quad 150$ 


\begin{tabular}{|c|c|c|c|c|c|c|c|c|}
\hline & NAME. & Size. & & Price o & of Plant & & Price of & Seeds. \\
\hline & & & Each & 10 & 100 & 1000 & Oz. & Lb. \\
\hline D t & Pawlonia imperialis & & & & & & & $\$ 075$ \\
\hline $\mathrm{E} t$ & Persea caroliniana-Red Bay & $--2-3 \mathrm{ft}$ & 8025 & $\$ 200$ & 1000 & & 25 & 150 \\
\hline & & $2-3$ it. col. & & 100 & 500 & & & \\
\hline $\mathrm{D} \mathrm{s}$ & Philadelphus coronarius - Mock Orange _.. & $-\ldots-1 \mathrm{ft}$ & 15 & 75 & 500 & & & \\
\hline Es & Phillyraea laurifolía & $-1_{2} \frac{1}{2} \mathrm{ft}$. & 35 & 250 & & & & \\
\hline E t & PINUS AUSTRALIS-Long-leaf Pine & -1-2 ft. root pruned & 50 & 300 & 2500 & 20000 & 45 & 275 \\
\hline $\mathrm{E} t$ & Pinus excelsa-Bhotan Pine & ... 15 - $18 \mathrm{in.}$ & 50 & 300 & 2000 & & & \\
\hline E t & " laricio corsíca-Corsican Pine _......... & - $12-18$ in. & 50 & 300 & 2000 & & 45 & 275 \\
\hline E t & mitis-Short-leaf Pine & _._. $1 \mathrm{ft}$. root pruned & $\tilde{5} 0$ & 300 & 2500 & & 45 & 275 \\
\hline $\mathrm{E} t$ & " taeda-Old Field Pine & ...1 ft. root pruned & 50 & 300 & 2500 & & 45 & 275 \\
\hline $\mathrm{D} \mathrm{t}$ & Platanus occidentalis - Sycamore & $-4-6 \mathrm{ft}$ & 25 & 120 & 800 & & 15 & 50 \\
\hline $\mathrm{E} \mathrm{t}$ & PRUNUS CAROLINIANA-Carolina Cherry & -_- $3-9$ in. $\mathrm{S}$. & & 40 & 250 & 1200 & & \\
\hline & & 12-18 in. & 10 & 50 & 300 & 2000 & & \\
\hline & & $18-24$ in. & 20 & 100 & 500 & 3000 & & \\
\hline & & $2-4 \mathrm{ft} . \mathrm{col}$ & & 75 & 500 & 3000 & 15 & 75 \\
\hline $\mathrm{Ds}$ & PYRUS ARBU'TIFOLIA-Chokeberry _...-. & $-\ldots-3 \mathrm{ft}$ & 25 & 100 & 300 & & 20 & 125 \\
\hline & $\begin{array}{c}\text { Our plants have RED not as commonly BLAC'K } \\
\text { berries. Much more ornamental. }\end{array}$ & 2-3 ft. col. & & 75 & 200 & 1500 & & \\
\hline $\mathrm{D} \mathrm{s}$ & Prunus tríloba-Double Almond & $----3-4 \mathrm{ft}$. & 25 & 200 & & & & \\
\hline $\mathrm{D} \mathrm{t}$ & Quercus alba-White Oak & .... 1-2 ft. col. & 15 & 100 & 400 & & & 20 \\
\hline E t & QUERCUS LAURIFOLIA - Water Oak _... & $\ldots-12-18$ in. & 20 & 100 & 500 & 3000 & *500 & 30 \\
\hline & & 3-5 ft. col. & 25 & 200 & 1800 & 15000 & & \\
\hline $\mathrm{Et}$ & DARLINGTON-1 & $\begin{array}{c}\text { Parlington Oak } \\
12-18 \text { in. }\end{array}$ & 20 & 100 & 500 & 3000 & $\div 500$ & 30 \\
\hline & & $3-5 \mathrm{ft} . \mathrm{col}$. & 30 & 250 & 2000 & 15000 & & \\
\hline $\mathrm{Dt}$ & PRINOS MONTICOLA- & $12-15$ in. $\mathrm{S}$. & 15 & 100 & 400 & & & 25 \\
\hline & Mountain Chestnut Oa & $\mathrm{k}_{-\ldots} \ldots 2-3 \mathrm{ft} . \mathrm{col}$. & 25 & 200 & 1800 & & & \\
\hline
\end{tabular}

*Bushel. 
NAME.
I) s Robinia hispida-Bristly Locust

E v Rosa bracteata-McCartney Rose

E r ROSA LAEVIGATA-Cherokee Rose

D s Rosa rugosa-Russian Rose

$\frac{1}{2} \mathrm{E}$ v ROSA WICHURAIANA_Japanese Trailing Rose_12-24 in.

D v ROSES-Deciduous Climbers, R. microphylla, Moss, Gem of the Prairies, Crimson Rambler, Yellow

Rambler, Empress of China, \&c., \&c.,_._._._-_.

${ }_{2}^{1} \mathrm{E}$ v Rubus hispidus-Swamp Blackberry_-_._-_._-_._-_1S-24 in.

Es Ruta graveolens-Rue

D t Salix babylonica-Weeping Willow

Es Santolina chamaecyparissus

D s Sassafras officinale

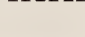

E $v$ SMILAX LAURIFOLIA-Laurel-leared China Brier

Size.

12 in.

3-ว็ ft. col.

-15-1s in. col. 6-9 in.

$1-2 \mathrm{ft}$. 6-9 in. $12-2+$ in. 9-12 in. $2-3 \mathrm{ft}$. 12 in.

\section{$3-4 \mathrm{ft}$.} 6-12 in. $-2-3 \mathrm{ft}$. $2-3 \mathrm{ft}$. col.
Price of Plants.

$\begin{array}{llr}\text { Each } \quad 10 \quad 100 & 0.57\end{array}$

$\$ 025 \$ 125 \$ 700$

$25 \quad 200 \quad 1800 \quad 15000$

$15 \quad 100 \quad 600 \quad 3000$

15 .75 $200 \quad 1200$

$25 \quad 100 \quad 400 \quad 2000$

$\begin{array}{lllll}15 & 60 & 200 & 1200\end{array}$

$20 \quad 75 \quad 300 \quad 2000$

$25 \quad 150 \quad 700$

$20 \quad 100 \quad 600 \quad 3000$

$30 \quad 2001000$

$15 \quad 120 \quad 400$

$15 \quad 50 \quad 300 \quad 1500$

$15 \quad 75 \quad 400 \quad 2500 \quad \$ 025$

25

$15 \quad$ S5 600

$25 \quad 150 \quad 600 \quad 3500 \quad 20$

$15 \quad 100 \quad 300 \quad 1200$

$3 \mathrm{ft}$. strong col. 2

D v "WALTERI-Walter's China Brier__-_._-3 ft. strong col.

D t Sophora japonica $-2-8 \mathrm{ft}$.

D s Spiraea prunifolia-Bridal Wreath $-1 \frac{1}{2}-2 \mathrm{ft}$.

$\mathrm{D} \mathrm{s}$

" thunbergii-Thunberg's Meadow Sweet 6-9 in.

D s Symphoricarpos vulgaris-Indian Currant $-2-2 \frac{1}{2} \mathrm{ft}$.

E t Taxodium distichum-Bald Cypress $2-2 \frac{1}{2} \mathrm{ft}$.

Price of Seeds. Oz. Lb. 


\section{NAME.}

D) t Tília americana-American Linn

D) $t$ Ulmus americana-Elm

E v VACCINIUM CRASSIFOLIUM-Creeping Huckieberry

F) VACCINIUM CRASSIFOLIUM-Creeping Huteklebert.

D s Viburnum nudum-Possum Haw-

D $s$

prunifolium-Black Haw

D v Vitis rotendifolia-Muscadine Grape

E $v$ Vinca major

E v VINCA MINOR-Periwinkle

D s Xanthorrhiza apiifolia-Yellow Root

E s YUCCA ALOIFOLIA-Spanish Bayonet
$2-3 \mathrm{ft}$.

2-3 ft. col. 9-12 in.

2-3 ft. col. 2 ft. col. strong. strong. 9-12 in.

1-2 ft. col. 1-2 ft.

$2-3 \mathrm{ft}$.

Price of Plants.

$\begin{array}{lll}\text { Each } & 10 \quad 100\end{array}$

$\$ 040 \$ 125$

$50 \quad 150$

20) $150 \quad 500$

$20 \quad 125 \quad+00$

25 $100 \quad 500$

$\begin{array}{llll}60 & 300 & 1500\end{array}$

$20 \quad 125 \quad 500$

$100+00$

$\begin{array}{lll}10 & 60 & 300\end{array}$

$10 \quad 50 \quad 200 \quad 800$

$15 \quad 100 \quad 250 \quad 800$

$15 \quad 75 \quad 400 \quad 1500$

$50 \quad 250 \quad 800$

$30 \quad 250$

$40 \quad 350$

3-5 ft. From .50 to 100 -tubers.

leaves $6-8 \mathrm{in.}$ long 15

10

" $8-12$ in.

largest plants

20

20
30

Price of Seed-

Oz. Lh.

$\$ 00 \$ 010 \$ 060$ 500

$25 \quad 100$

$15 \quad 75$

$15 \quad 75$

25125

$30 \quad 125$

45

$40 \quad 250$

PINEHURST VIOLET Clumps

$\begin{array}{llll}10 & 75 & 300 & 2500\end{array}$ 


\section{FRUIT DEPARTMENT.}

NAMF,

GRAPEVINES-Delaware, Moore's Diamond, Moore's

Early, Niagara, Catawba

We wish to discontinue the propagation of grapevines. Our

stock of about 20,000 vines is in grand condition and a rare

bargain at above prices.

CORDONS-Single and double arm 3-5 $\mathrm{ft}$.

250

APPLES:-(on Paradise) Alexander, Baldwin, Chenango, Early Harvest, Gravenstein, Jonathan, Northern Spy, Primate, Sweet Bough, Tompkins King, Wagener, Wealthy.

PEARS:-(on Quince) Angouleme, Anjou, Bartlett, Clapp's Favorite, Easter Beurre, Hardy Giffard, Howell, Josephine of Malines, Lawrence, Louise Bonne of Jersey, Superfin.

These peculiarly trained dwarf fruit trees, of which we have to offer about one hundred, are especially adapted to private places where they surely will be a permanent source of interest, pleasure and profit. Year in year out they will repay diligent care by sure crops of fruits of extra large size and of a very superior quality. The main trunk of our about three year old plants is bent 18 inch above ground in " $T$ " shape or with a few with. one arm. Our trees are covered with fruit-branchlets and are ready to bear next year. We are ready to give any desired cultuxal directions. 


\section{HERBACEOUS PLANTS.}

A, Annuals. P, Per̈ennials. B, Bog Plants and Aquatics. F, Ferns. O, Orchids.

NAME.

Adiantum pedatum-Maidenhair

P Aletris farinosa-Unicorn Root

Arenaria diffusa-Sandwort

Asarum virginicum-Snakeroot

ASCLEPIAS TUBEROSA-Butterfly Weed

Asperula azurea-Blue-flowered Forest Master

Asplenium ebeneum-Spleenwood

Aspidium acrostichoides

Baptisia tínctoria-IVild Indigo

Breweria pickeringii

Chimaphila maculata-Prince's Pine

Chrysopsis graminifolia-Scurvy Grass

CLITORIA MARIANA-Butterfly Pea

Cirsium repandum-Scotch Thistle

Coreopsis aurea-Golden Tickweed

" lanceolata

lanceolata angustifolia

Crotalaria sagittalis-Rattle-box

Ctenium americanum-Toothache Grass

Cypripedium acaule - Lady's Slipper

pubescens

Datura stramonium-Thorn Apple

Dicentra eximia-Bleeding Heart.
Price of Plants.

$10 \quad 100 \quad 1000$

$\$ 050$

$50 \$ 150 \$ 1000$

$\begin{array}{lll}100 & 500 \quad 3000\end{array}$

$\begin{array}{llll}60 & 200 & 1500\end{array}$

$80 \quad 400 \quad 2500$

$50 \quad 300$

$50 \quad 200$

$100 \quad 400$

$75 \quad 300$

$100+00$

$100 \quad 500$

$75 \quad 300$

$100 \quad 400$

$60 \quad 250$

$100 \quad 400$

100

100

400

300

150

150
Price of Seeds.

Plkt. Oz.

$\$ 010 \$ 030$

$10 \quad 40$

$10 \quad 100$

$10 \quad 50$

$10 \quad 25$

05

25

20

$10 \quad 50$

$10 \quad 30$

$25 \quad 60$

10

$10 \quad 50$

$10 \quad 50$

$10 \quad 50$

$10 \quad 50$

$10 \quad 50$

10 
NAME.

DIONAEA MUSCIPULA-Venus Flytrap

B Drosera rotundifolia-Round-leaved Sundew EPIGAEA REPENS-Mavflower

Eriocaulon decangulare-Pipewort

Eriophorum virginicum-Cotton Grass

GALACTIA GLABELLA-Nilk Pea

Galax aphylla

Gentiana saponaria-Soapwort Gentian

Gerardia purpurea-False Foxglove

Gillenia stipulacea-Indian Physic

o Habenaria ciliaris

Helleborus hybridus

HEUCHERA AMERICANA

Impomoea pandurata-Wild Potato Vine

IRIS CRISTATA

Iris pallida, dark blue

" verna

"versicolor-American Blue Flag

JATROPHA S'TIMULOSA-Spurge Nettle

Liatris graminifolia-Button Snake Root

" squarrosa-Blazing Star

" tenuifolia

Lilium superbum-Turk Cap's Lily

LUPINUS DIFFUSUS-Deer Cabbage

Mimulus ringens-Monkey Flower

Nymphaea odorata-Water Lily

Opuntia vulgaris-Hardy Cactus
Price of Plants.

$10 \quad 100 \quad 1000$

$\$ 075 \$ 350 \$ 2500$

$50 \quad 200$

$75 \quad 300$

$\begin{array}{lll}80 & 300 & 2000\end{array}$

$100 \quad 400$

$60 \quad 300$

$\begin{array}{ll}75 & 400\end{array}$

$100+00$

$\begin{array}{lll}85 & 400 \quad 1800\end{array}$

$75 \quad 500$

$75 \quad 300 \quad 2000$

50 each.

125600

$85 \quad 700$

$50 \quad 300$

250

.30 each

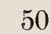

300

1200

$\begin{array}{ll}75 & 200\end{array}$

$\begin{array}{lll}100 & 300 \quad 1500\end{array}$

$\begin{array}{lll}50 & 200 & 1200\end{array}$

$\begin{array}{lll}50 & 200 \quad 1000\end{array}$

$\begin{array}{lll}50 & 200 & 1000\end{array}$

$72+00$

$50 \quad 300$

1800

$\begin{array}{lll}50 & 200 & 1200\end{array}$

$100 \quad 500$

$50 \quad 200$
Price of Seeds.

Pkt. Oz.

$\$ 025 \$ 200$

$10 \quad 50$

$05 \quad 20$

10

10

40

$10 \quad 30$

$10 \quad 20$

$10 \quad 20$

$05 \quad 20$

$10 \quad 50$

05

05 
XAME.

B ORONTIUM AQUATICUM-Crolden Club

F Osmunda cinnamomea-Cimnamon Ferin

Passiflora incarnata-Passiontiotrer

PHLOX SUBULATA_Moss Pink

Polygala luitea

Pogonía divaricata

ophioglossoides

PYXIDANTHERA BARBULATA-Flowering Moss, Pyxie

Rhexia mariana-Deer Grass

" vírginiana-Meadow Beauty.

Rhynchosia erecta reniformis

- SABBATIA BRACHIATA-American Centaury

B Sagittaria variabilis-Arrow Leaf

Sanguinaria canadensis-Blood Root

Sarracenia flava-Trumpet Leaf

$$
\text { " }
$$

"4

purpurea-Pitcher Plant

variolaris-Spotted Trumpet Leaf

Schrankia angustata-Sensitive Plant.

Sericocarpos conyzoides-White-topped Aster

Silene virginica-Fire Pink

Sisyrrhinchium bermudianum-Blue-eyed Grass

Stillingia sylvatica-Queen's Delight

Stylosanthes elatior-Pencil Flower.

TEPHROSIA VIRGINICA-Devil's shoe String

Tofieldia pubens-Downy False Asphodel

P Tradescantia rosea-Rose Spiderwort
Price of Plants.

$10 \quad 100 \quad 1000$

Price of Seeds

Pkt. Oz.

\section{\$0 60 8. 00}

$100+00 \$ 2500$

$50 \quad 300$

$\$ 0 \quad 10 \quad 8030$

50 $300 \quad 2000$

$50 \quad 250 \quad 1000$

$100 \quad 500$

$100 \quad 500$

100400

$75 \quad 300$

$50 \quad 250$

1500

$05 \quad 30$

$05 \quad 30$

$60-300-1000$

$05 \quad 40$

$50 \quad 200 \quad 1000$

$05 \quad 40$

$10 \quad 60$

70300

$125 \quad 600$

$100+00$

$100 \quad 300$

2500

$10 \quad 60$

$10 \quad 60$

$10 \quad 60$

$20 \quad 60$

$100 \quad 400$

2000

$100 \quad 400$

$75 \quad 300$

$75 \quad 250$

1200

$10 \quad 50$

$10 \quad 50$

$10 \quad 30$

$10 \quad 50$

$10 \quad 75$ 


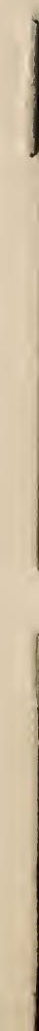


To enable our patrons to make their selections more readily, we have compiled the following lists, which we trust will be found of good use. For prices look up names in their respective places in alphabetical list.

\section{SHADE TREES.}

Acer rubrum saccharinum

Albizzia julibrissin

Diospyros virginiana

Fagus ferruginea

Liquidambar styraciflua

Cedrus atlantica

"6 deodara

Cephalotaxus fortunei

Chamaecyparis thuyoides

Berberis thunbergi

"6 vulgaris

Buxus sempervirens

Chamaecyparis thuyoides

Cliftonia ligustrina

Cotoneaster microphylla

Euonymus radicans var

Gelsemium sempervirens

Hedera helix

$1 / 2 \mathrm{E}$ Akebia quinata

Gelsemium sempervirens Hedra helix

Ampelopsis quinquefolia Bignonia capreolata
Liriodendron tulipifera

Magnolia acuminata

Melia azederach

Melia umbraculifera

Nyssa sylvatica

Platanus occidentalis

Prunus caroliniana

Quercus alba

II. CONIFERS.

Cryptomeria japonica

Cupressus fastigiata

Juniperus virginiana
" grandiflora

" tripetala

\section{HEDGE PLANTS.}

\section{EVERGREEN CLIMBERS.}

Lonicera brach. halleana Lonicera sinensis

"fol. aureo reticulatis Rosa bracteata

\section{DECIDUOUS CLIVIBERS,}

Bignonia radicans

Quercus laurifolia

" darlington

" monticola

Sophora japonica

Tilia americana

Pinus mitis

" taeda

Taxodium distichum
Cupressus lawsoniana

Cydonia japonica

Euonymus japonica

Gardenia florida

Ilex glabra

" opaca

Ligustrum chinense nanum

" ovalifolium

Ilex dahoon

\section{GROUND-COVERING PLANTS.}

Hypericum calycinum

" moserianum

Lonicera halleana

Rosa rugosa

Rosa wichuraiana

Rubus hispidus

Santolina chamaecyparissus

Vaccinium crassifolium
Limonia trifoliata Maclura aurantiaca Prunus caroliniana Spiraea thumbergii

Rosa laevigata

Smilax laurifolia

ROSES: Crimson Rambler Yellow Rambler Prairie Gem, \&c.

Smilax walteri

VII. DECIDUOUS TREES AND SHRUBS OF EVERGREEN ASPECT.

Euonymus americana Genista all.

Limonia trifoliata

Sophora japonica 
